\title{
REAL-TIME POWERLINE CORRIDOR INSPECTION BY EDGE COMPUTING OF UAV LIDAR DATA
}

\author{
S. Pu ${ }^{1, *}$, L. Xie ${ }^{2}$, M. Ji ${ }^{1}$, Y. Zhao ${ }^{1}$, W. Liu ${ }^{2}$, L. Wang ${ }^{2}$, Y. Zhao ${ }^{2}$, F. Yang ${ }^{2}$, D. Qiu ${ }^{2}$ \\ ${ }^{1}$ Beijing Tovos Technology Co., Ltd, Zhongguancun Nanadjie 32, 100081 Beijing, China - (pushi, zhaoyongyi, jimingru)@ tovos.net \\ ${ }^{2}$ Guangdong Power Grid Co., Ltd, Dongfengdonglu No.757, 510060 Guangzhou, China - (longguangxie, wensongliu, leiwang, \\ yongqiangzhao, fangyang, danqiu) @ gd.csg.cn
}

Commission II, WG II/4

KEY WORDS: Powerline Inspection, UAV LiDAR, Edge Computing

\begin{abstract}
:
This paper presents an innovative power line corridor inspection approach using UAV LiDAR edge computing and 4G real-time transmission. First, sample point clouds of power towers are manually classified and decomposed into components according to five mainstream tower types: T type, V type, $\mathrm{n}$ type, I type and owl head type. A deep learning AI agent, named "Tovos Agent" internally, is trained by supervised deep learning the sample data sets under a 3D CNN framework. Second, laser points of power line corridors are simultaneously classified into Ground, Vegetation, Tower, Cable, and Building types using semantic feature constraints during the UAV-borne LiDAR acquisition process, and then tower types are further recognized by Tovos Agent for strain span separation. Spatial and topological relations between Cable points and other types are analyzed according to industry standards to identify potential risks at the same time. Finally, all potential risks are organized as industry standard reports and transmitted onto central server via $4 \mathrm{G}$ data link, so that maintenance personal can be notified the risks as soon as possible. Tests on LiDAR data of 1000KV power line show the promising results of the proposed method.
\end{abstract}

\section{BACKGROUND}

As a rapid developing country, China holds an increasing demands for power consumption over decades. As a result, more and more transmission power lines has been constructed to support a larger and more stable power grid. At the end of 2018 there are over 140 million kilometres' transmission power line all over China, which are almost half of the world's overall transmission power lines. However, the traditional power line inspection methods are rather manual, slow and dangerous. Inspection personals have to walk along the power line corridors, visually identify the potential risks such as high vegetation, overstretched cables and illegal infrastructures, and then evaluate the risk levels from their own experience. In order to overcome the disadvantages of manual visual inspection, helicopter-borne laser scanning has been introduced in China since 2014. However, the improvement has been considered trivial because of the expensive cost of helicopters and the lacking of automated safety inspection programs from laser points. Power grid industry is still seeking for more efficient and more intelligent power line corridor inspection methods.

There are three main groups of corridor risks. They are:

- Physical risks, such as partially broken cables and damages on insulators.

- Clearances risks, such as insufficient clearance distances between cables and vegetation.

- Temperature risks, such as extreme temperatures of power line facilities.

The physical risks are usually captured by taking several images from different angles during UAV or helicopter inspection, and then manually recognized by human operator or automatically detected by image processing methods. However, at this time manual recognition of physical risks is still the primary approach, because there are no automatic software yet which provides reliable and stable recognition results. The temperatures risks can be easily extracted from thermal images taken from airborne inspection, so they are not discussed in this paper.

Obviously it is impossible to detect clearance risks from single image, therefore it is necessary to acquire three dimensional data provided by either laser scanning or oblique photogrammetry. The advantage of oblique photogrammetry is its low cost, since image sensors are still much cheaper than LiDAR sensors. However, the application of oblique photogrammetry on power line inspection is not successful and has been abandoned gradually after a few years' attempts in China. This is because first, power line cables are thin features instead of planar features, so that corresponding matches can hardly be identified during image matching; second, the background of power line corridors are rather monotonous since they are usually built in desolate regions, which also result in difficult image matching. It seems laser scanning is the most appropriate choice for detection of clearance risks. As the rapid drop of short range laser sensor's price, laser scanning have been more and more popular in China for power line inspection.

The rapid development of UAV techniques also promoted the application of UAVs in power line inspections. The government policies for helicopter operation is very strict in China, as a result it is very expensive and tedious to inspect power line corridors by helicopter. Under such circumstances, UAV has been chosen as an alternative data acquisition platform naturally. The UAVs are usually operated by power line inspectors of the power grid companies. They are not trained with professional survey and mapping knowledge, so the equipped laser scanning devices must be easy to use, and the data processing procedure should be

\footnotetext{
${ }^{*}$ Corresponding author
} 
as simple as possible. The price and range of UAV-based laser systems should also match the price and flying height of UAV, since it is not acceptable in power grid industry to mount a laser system cost hundreds of thousands of Euros on UAVs cost only thousands of Euros.

This paper presents an innovative power line corridor inspection approach using UAV LiDAR edge computing and 4G real-time transmission. Section 2 introduces the overall methodology of our work. Section 3 provides the hardware design of our UAVbased powerline inspection system. Section 4 explains the data processing algorithms. Test cases and results are given in section 5. Concluding remarks and outlooks are given in Section 6.

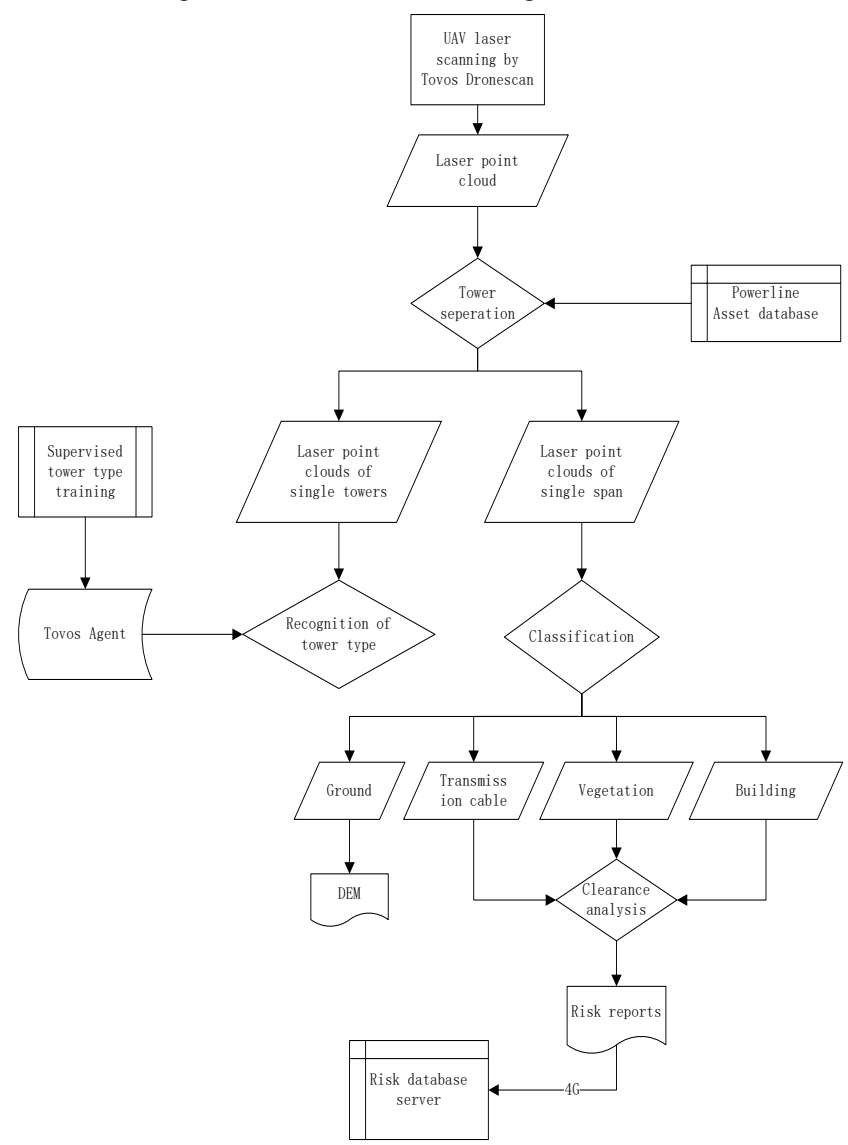

Figure 1. Data flow framework

\section{THE METHODOLOGY}

Figure 1 illustrates the overall data flow framework of our methodology. First, sample point clouds of power towers are manually classified and decomposed into components of tower base, body, cross arm and head. A deep learning AI agent, named "Tovos Agent" internally, is trained by supervised deep learning the sample data sets under a 3D CNN framework (Hansen and Salamon 1990). Second, laser points of power line corridors are simultaneously classified into Ground, Vegetation, Tower, Cable, and Building types using semantic feature constraints during the UAV-borne LiDAR acquisition process, and then tower types are further recognized by Tovos Agent for strain span separation. Spatial and topological relations between Cable points and other types are analyzed according to industry standards to identify potential risks at the same time. Finally, all potential risks are organized as industry standard reports and transmitted onto central server via $4 \mathrm{G}$ data link, so that maintenance personal can be notified the risks as soon as possible.

\section{HARDWARE DESIGN}

In order to fulfill the requirement of real-time UAV laser scanning and risk analysis promoted by the Power Grid customers, a light-weight LiDAR system named Tovos DroneScan is designed and has been operational since May 2018 (see Figure 2 for exterior appearance). Some key technical parameters of Tovos DroneScan is given in Table 1. The system consists of both LiDAR and optical sensors. Relative orientation has been calibrated during manufacture so that colored point cloud can be generated. The DJI M600 pro is chosen as the primary UAV mounting platform for Tovos DroneScan due to its excellent control performance and low cost, but it should be mentioned that UAV performs only as a mechanic mounting platform, with no electric or data connection with the LiDAR system.

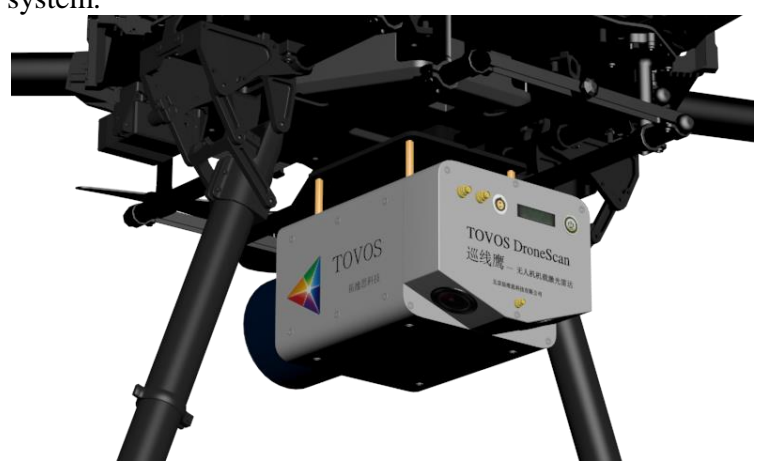

Figure 2. Tovos DroneScan mounted on DJI M600 pro

\begin{tabular}{|l|l|}
\hline \multirow{4}{*}{ LiDAR sensor } & Range: $100 \mathrm{~m} / 150 \mathrm{~m} / 200 \mathrm{~m}$ \\
\cline { 2 - 2 } & Laser beams: $16 / 32 / 40$ \\
\cline { 2 - 2 } & Accuracy: $5 \mathrm{~cm}$ \\
\cline { 2 - 2 } & FOV: 360 degree \\
\hline Optical sensor & 10 Million pixels * 2 , stereo aligned \\
\hline \multirow{2}{*}{ GNSS module } & Position accuracy: 0.03 cm (RTK) \\
\cline { 2 - 2 } & Roll/Pitch accuracy: 0.035 degree \\
\cline { 2 - 2 } On-board & Heading accuracy: 0.15 degree \\
computer & $\begin{array}{l}\text { CPU: i7 5500u; } 8 \mathrm{G} \text { memory; } 120 \mathrm{G} \\
\text { hard disk }\end{array}$ \\
\hline Wireless module & $\begin{array}{l}\text { TDD-LTE/WCDMA; 300Mbps } \\
\text { downlink/50 Mbps uplink }\end{array}$ \\
\hline Weight & 3.1 Kg \\
\hline UAV platform & DJI M600 pro or equivalent \\
\hline
\end{tabular}

Table 1. Tovos DroneScan technical parameter

\section{DATA PROCESSING}

The data processing procedure consists of two stages: a "inflight" processing stage in which only the vital vegetation risks are identified while UAV flying, and a "post-flight" processing stage in which more environment types are classified and more regular risks are identified after the LiDAR data are downloaded from Tovos DroneScan to a regular computer.

\subsection{Calculation of laser points}

The output laser points of Tovos DroneScan is in WGS 84 coordinate system. The calculation of coordinates requires three kinds of input data:

- Local laser points provided by the LiDAR sensor;

- Global positions and orientations provided by the GNSS module;

- Image sequences provided by the optical sensors, as well as their interior and exterior parameters. 
The calculation procedure is automatically done during flying and is blind to the human user. It should be noted that the data rate of LiDAR sensor is much faster than the GNSS data, so certain interpolation of positions and orientations are applied. The absolute accuracy of the output point cloud is $5-10 \mathrm{~cm}$, which is lower than post processing in professional software such as Novatel Inertial Explorer. But its more cost-effective to calculate laser points in real-time and the accuracy is acceptable for powerline inspection.

\subsection{Recognizing tower types}

The locations of power line towers can be retrieved from China State Grid's asset database, while the tower types are not reliable in these databases and should be recognized from actual LiDAR point clouds. The purposes of recognizing tower types are: 1) tower types are important to separate strain spans, which are the basic region for risk assessment, and 2) tower types can be used by parameterized modeling method to generate $3 \mathrm{D}$ wireframe models automatically.

There are five mainstream tower types: $\mathrm{T}$ type, $\mathrm{V}$ type, $\mathrm{n}$ type, I type and owl head type. Over 300 sample laser point clouds of different power line corridors are manually decomposed into tower base, body, cross arm and head. See Figure 3 for the composition of a T type tower. A deep learning AI agent, named "Tovos Agent" internally, is trained by supervised deep learning the sample data sets under a 3D CNN framework. During actual tower type recognition, the point cloud of each tower is extracted from the whole corridor based on tower locations and a radius, the Tovos Agent is applied to identify a type with best alpha shape match.
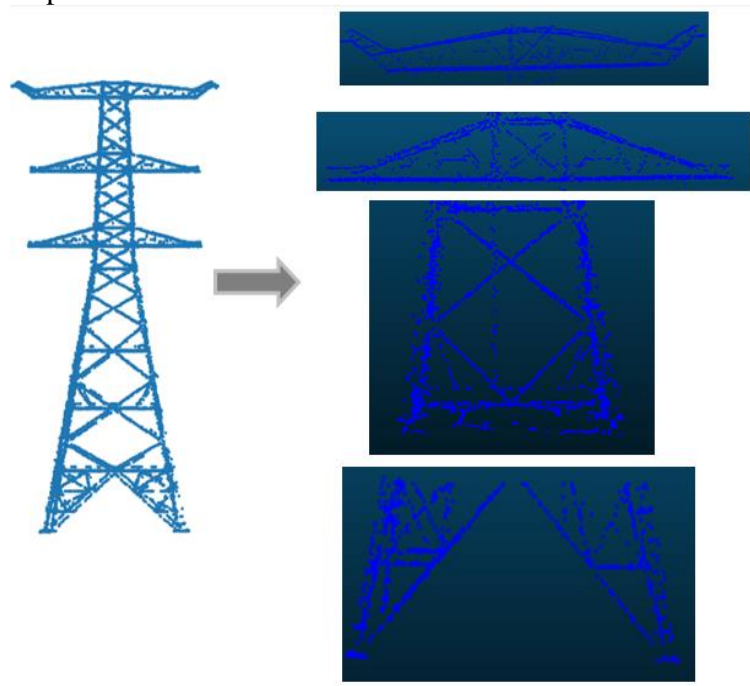

Figure 3. Decomposition of a T type tower

\subsection{Corridor classification}

There are several feature types within a power line corridor, such as Ground, Vegetation, Tower, Transmission cable, Ground cable, Building, Road and Low-level power line. So far our automatic classification algorithm can extract Ground, Cable, Tower, Vegetation and Building reliably, which are also the most concerned features for risk analysis. Less significant features such as Road and Low-level power line are manually recognized after UAV flight stage. Towers are extracted using their locations from asset database, as mentioned in 4.2. The recognition of Ground, Vegetation, Building and Cable features are similar to the semantic feature recognition method in $\mathrm{Pu}$ and Vosselman (2009). The method is explained as follows:
1) Using progressive TIN densification (Zhang and Lin, 2013) to recognize Ground points.

2) Segment the rest points using surface growing algorithm (Vosselman et al., 2004).

3) Analyze the location, area, orientation of the laser segments. Cable feature is considered parallel with the corridor direction, and can be fitted to lines in horizontal plane. Building feature are recognized from strong planar roofs, and should have regular roof shapes.

4) All the sparse distributed points are assigned as Vegetation type. Indeed there are some noise points such as birds or ground poles recognized as vegetation in this way, but these mistakes can be easily identified in the final risk report and corrected.

\subsection{Analysis of potential risks}

Definition of power line corridor risks cables in different voltage levels have been strictly regulated in power industry. The risks concerns the distances and angles between corridor elements and the cables, and the changes of the corridor elements. Table 2 gives an example of the clearance regulation published by China State Grid. To analyze potential risk, laser points of each strain span are spatially indexed with Octree, then each Cable point is compared with other laser segment to check the violation of horizontal, vertical and three dimensional distances and angles.

\begin{tabular}{|l|l|l|}
\hline Corridor element & $\begin{array}{l}\text { Horizontal } \\
\text { distance (m) }\end{array}$ & $\begin{array}{l}\text { Vertical distance } \\
(\mathbf{m})\end{array}$ \\
\hline Ground & & 19 \\
\hline Building & 7 & 17.5 \\
\hline Vegetation & 13.5 & 13.5 \\
\hline Road & & 21.5 \\
\hline Railroad & & 21.5 \\
\hline River & & 15 \\
\hline $\begin{array}{l}\text { Low-level power } \\
\text { line }\end{array}$ & & 10.5 \\
\hline
\end{tabular}

Table 2. Distance regulation published by China State Grid

Standard <<GBT28813-2012 $\pm 800 \mathrm{KV}>>($ GBT, 2012)

\section{TEST CASES}

In total over 3000 kilometers' power line corridors in China have been laser scanned using Tovos Dronescan. Figure 4 demonstrates the inspection procedure of a single power line span of $1000 \mathrm{KV}$ owned by China State Grid. The UAV flight of this data was finished in October 2018. The UAV flight trajectory is almost parallel with the center line of the power line corridor's direction, and the flight altitude is about 15 meters above the ground cable. The flight speed is 5 meter per second in average. The point cloud of the span is 550 meters' long, and was cut to be 70 meters wide, with center axis coincide with line defined by the two tower centers.

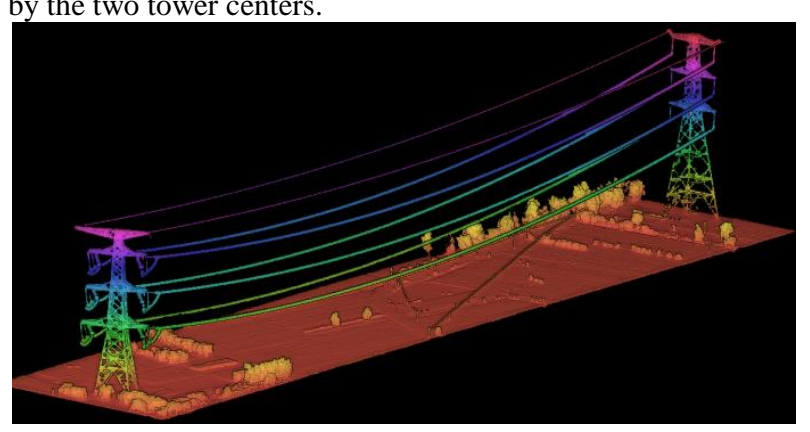




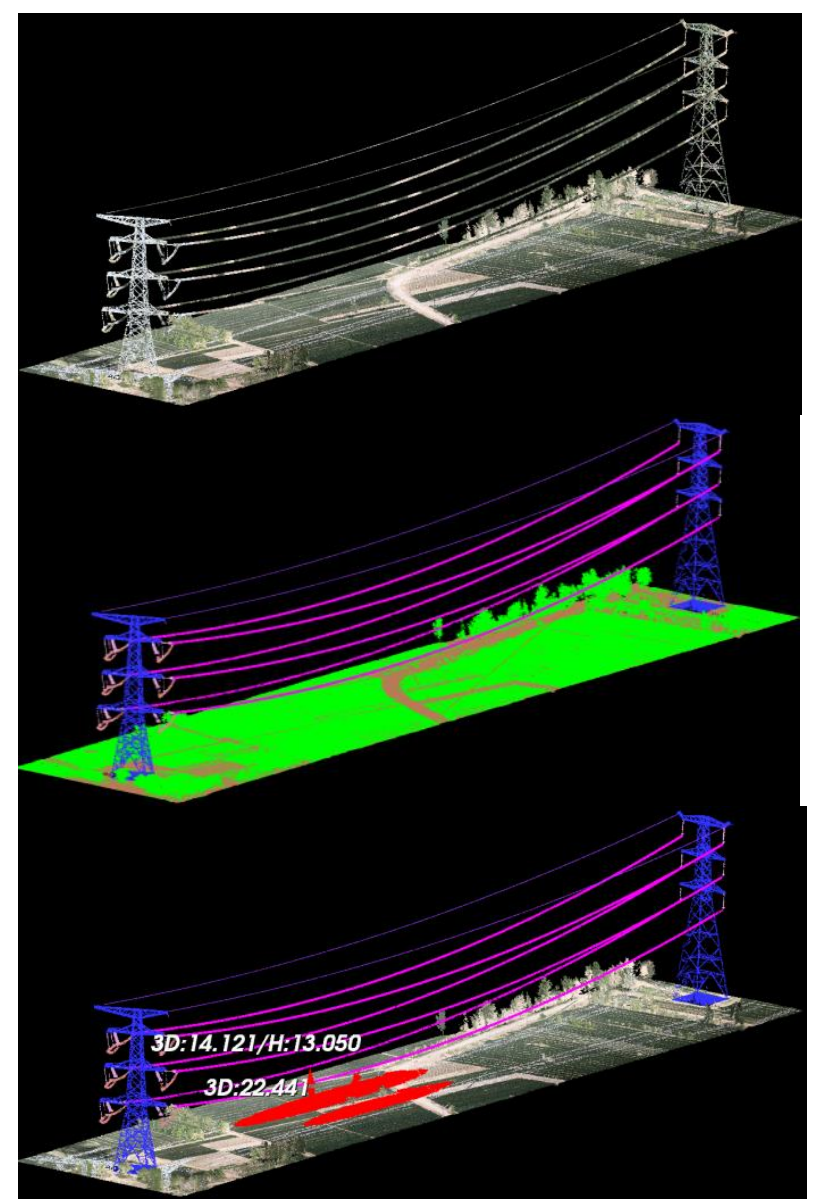

Figure 4. Test result of a $1000 \mathrm{KV}$ power line span (from top to bottom: original laser point cloud; colored point cloud; after classification; highlighted risk regions)
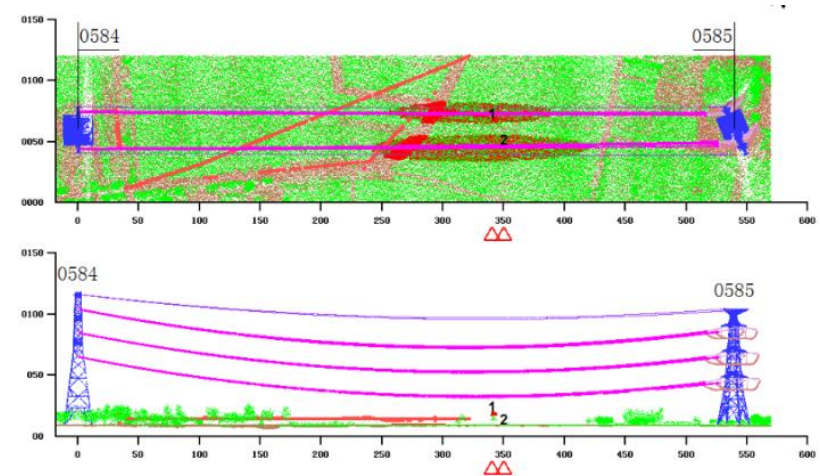

Figure 5. Part of a risk report (up: top view of a power line corridor; down: front view)

The whole point cloud contains $3,274,598$ points in total, with a average point density of 110 points per square meter on ground. It took 194 seconds to finish all the data analysis procedure. A time counter is inserted in the program to tag the time spent on each data processing steps, and the result is shown in Table 3. The classification step obviously took a long time, probably due to the calculation of TIN and point cloud segmentation. The calculation should be faster if better on-board computers are used, but the improvement becomes less significant if the increase in hardware cost and size are taken into consideration. At this time we have no optimal solution to accelerate these calculations. Since the data analysis procedure took much longer than the data acquisition procedure, the Tovos Dronescan was kept still on the ground for several minutes after the UAV was landed, until report uploading via $4 \mathrm{G}$ was completely finished.

Test result in Figure 4 shows that our method produces satisfactory risk assessment result for power line safety inspection. Two regions (labeled red in Figure 4 bottom) violating industry clearance standards were identified. A webbased risk report was automatically generated (see Figure 5) and pushed directly from field flight to State Grid risk database. The analysis of tower types, colored point cloud generation, and DEM generation were actually finished later on using a regular PC instead of during UAV flying, because they are optional processing steps and are time consuming to apply in real time.

\begin{tabular}{|l|c|}
\hline Data processing step & Time (seconds) \\
\hline Coordinate computation & 26 \\
\hline Tower separation & 5 \\
\hline Classification & 131 \\
\hline Clearance analysis & 12 \\
\hline Risk report generation & 17 \\
\hline Report upload & 3 \\
\hline \multicolumn{1}{|c|}{ Total } & 194 \\
\hline
\end{tabular}

Table 3. Method efficiency evaluation result

\section{CONCLUDING REMARKS}

This paper presents a real-time power line corridor inspection approach using edge-computing device and methods. Both LiDAR data acquisition and analysis are executed during UAV LiDAR flight, and risk assessment reports are transmitted to central server via $4 \mathrm{G}$ network, so that power line maintenance personals could be notified in a shortest possible period and make necessary reaction. The future work will make attempt to transmit all the original data, such as LiDAR sensor data and IMU data, directly to background server via $5 \mathrm{G}$ network, so as to apply all the computation processes by much faster parallel computer clusters.

\section{ACKNOWLEDGEMENT}

This research is sponsored by Guangdong Power Gird Co., Ltd (Science Project No. GDKJXM20184286(036500KK52180006))

\section{REFERENCES}

GB/T, 2012. Operation Code for 800KV DC Overhead Transmission Line, in GB/T 28813-2012, ICS 29.240.20.

Hansen L K, Salamon P.,1990. Neural Network Ensembles. IEEE Transactions on Pattern Analysis and Machine Intelligence, 12(10), 993-1001.

Pu, S., Vosselman, G., 2009. Knowledge Based Reconstruction of Building Models from Terrestrial Laser Scanning Data, ISPRS Journal of Photogrammetry and Romote Sensing, 64, 575-584

Vosselman, G., B. Gorte, G. Sithole and Rabbani, T., 2004. Recognizing Structure in Laser Scanner Point Clouds. International Archives of Photogrammetry, Remote Sensing and Spatial Information Sciences, vol. 46, part 8/W2, Freiburg, Germany, October 4-6, 33-38.

Zhang J, Lin X., 2013. Filtering Airborne LiDAR Data by Embedding Smoothness-constrained Segmentation in 
The International Archives of the Photogrammetry, Remote Sensing and Spatial Information Sciences, Volume XLII-2/W13, 2019 ISPRS Geospatial Week 2019, 10-14 June 2019, Enschede, The Netherlands

Progressive TIN Densification. ISPRS Journal of

Photogrammetry and Remote Sensing, 81, 44-59. 